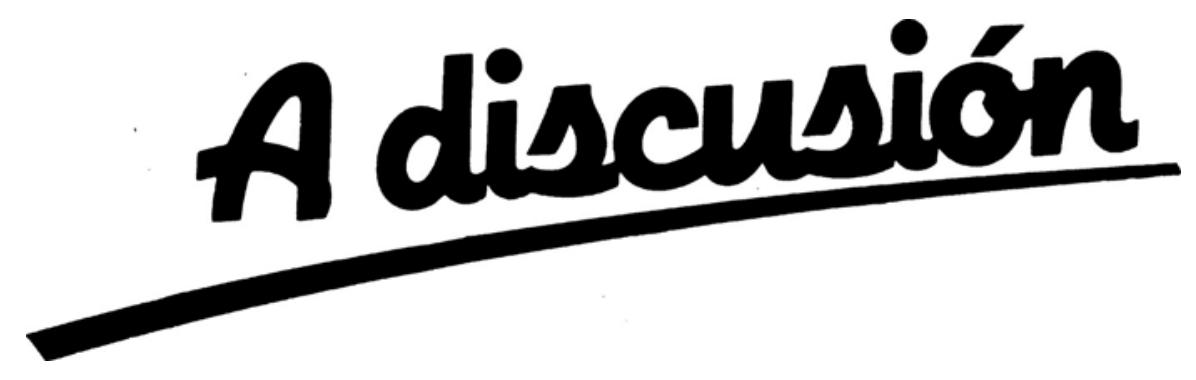

\title{
INVESTMENT OPTION UNDER CIR INTEREST RATES*
}

\section{Julio Carmona and Angel León**}

\author{
WP-AD 2007-24
}

Corresponding author: Angel León Valle, Dpto. Economia Financiera, Universidad de Alicante, San Vicente del Raspeig; 03080, Alicante, Spain. Tel: +3496590 3621, Fax: +34965903611. E-mail:aleon@ua.es.

Editor: Instituto Valenciano de Investigaciones Económicas, S.A.

Primera Edición Octubre 2007

Depósito Legal: V-4642-2007

Ivie working papers offer in advance the results of economic research under way in order to encourage a discussion process before sending them to scientific journals for their final publication.

* Financial support from the Spanish Ministry of Education and Science through the grant SEJ 200509372 (Leon) and SEJ 2004-05815 (Carmona) is gratefully acknowledged. The contents of this paper are the sole responsibility of the authors

** University of Alicante. 


\title{
INVESTMENT OPTION UNDER CIR INTEREST RATES
}

\section{Julio Carmona and Angel León}

\begin{abstract}
We analyze extensively the characteristics of the solution to an irreversible investment decision when the only source of uncertainty comes from interest rates. They are assumed to be driven by the popular Cox-Ingersoll-Ross (CIR) stochastic process. Particular attention is paid to the impact that both CIR parameters and risk aversion have on the threshold rate.
\end{abstract}

Keywords: CIR process, Project value, Real options, Risk aversion.

JEL Classification: C61, G31 


\section{Introduction}

The real option literature dealing with the effect of uncertainty on the value of (partially) irreversible investment projects is extensive and has been growing steadily from the seminal work of McDonald and Siegel (1986), among others. Ingersoll and Ross (1992) were the first in extending this analysis to uncertain interest rates. They analyze extensively the case in which interest rates follow a martingale process to describe their impact on the timing of investment. A recent paper by Alvarez and Koskela (2006a) considers decisions about irreversible investments under uncertainty in the interest rates and in the flow of revenues. Unfortunately, their generalized optimal stopping problem can only be solved numerically.

In our paper, we assume that interest rates are driven by the CIR process, popularized by Cox, Ingersoll and Ross (1985), and keep them as the only source of uncertainty. In this way, we are able to obtain a closed form solution to our easier optimal stopping time problem.

We also undertake a sensitivity analysis of how changes in the underlying parameters in the CIR process affect the optimal investment decision. Finally, we also study how changes in the degree of risk aversion affect the optimal stopping time, following the analysis of Alvarez and Koskela (2006b). In our approach, we focus instead on the market price of risk, which is more convenient given our setup. We show the equivalence between both approaches and extend our previous sensitivity analysis accordingly.

The rest of this paper is organized as follows. Section 2 provides the valuation of an investment project with the option to wait under the risk neutral measure. Section 3 performs an extensive sensitive analysis about how the threshold rate, obtained as part of the solution in the previous section, changes under alternative values of the parameters describing the CIR process. Section 4 performs the analysis 
for the impact of the market price of risk indicated above and finally, Section 5 concludes.

\section{Valuing the Option to Invest}

We are interested in the present value (PV) of an investment project that yields an expected continuous payoff of $\$ 1$ during the time $t$ through $t+T$. The interest rate $r$ is assumed, under the real measure $\mathbb{P}$, to follow the CIR process

$$
d r=\kappa(\bar{r}-r) d t+\sigma \sqrt{r} d W^{\mathbb{P}}
$$

where $\kappa>0$ is the mean-reversion coefficient, $\bar{r}$ is a target interest rate, $\sigma$ is the volatility and $d W^{\mathbb{P}}$ is the Wiener process under the real measure. However, for valuation purposes, it is much more convenient to define a new Wiener process under the risk neutral measure, denoted by $\mathbb{Q}$. Under this measure, $r$ evolves as

$$
d r=(\kappa+\lambda)\left(\frac{\kappa}{\kappa+\lambda} \bar{r}-r\right) d t+\sigma \sqrt{r} d W^{\mathbb{Q}}
$$

By Girsanov Theorem the correspondence between the two Wiener processes is given by

$$
d W^{\mathbb{P}}=d W^{\mathbb{Q}}-\gamma(r, t) d t
$$

where $\gamma(r, t)$ denotes the market price of risk that is defined for the CIR process as

$$
\gamma(r, t) \equiv-\frac{\lambda \sqrt{r}}{\sigma}
$$

The present value at time $t$ of the stream of cash-flows is given by

$$
V\left(r_{t}, t, T\right)=E_{t}^{\mathbb{Q}}\left[\int_{t}^{t+T} \exp \left\{\int_{t}^{s} r_{u} d u\right\} d s\right]=\int_{t}^{t+T} P\left(r_{t}, t, s\right) d s
$$


where $P\left(r_{t}, t, s\right)$ denotes the price at time $t$ of a zero-coupon bond maturing at time $s>t$ conditioned to the actual value $r_{t}$ of the stochastic interest rate, and it satisfies the following partial differential equation (PDE):

$$
\frac{\partial P}{\partial t}+\frac{\partial P}{\partial r}[\kappa(\bar{r}-r)-\lambda r]+\frac{1}{2} \frac{\partial^{2} P}{\partial r^{2}} \sigma^{2} r=r P
$$

with terminal condition $P(s, s)=1$. Its solution, as can be seen in Cox, Ingersoll and Ross (1985), is given by

$$
P\left(r_{t}, t, s\right)=A(t, s) e^{-B(t, s) r_{t}}
$$

where

$$
A(t, s)=\left(\frac{\theta_{1} e^{\theta_{2}(s-t)}}{\theta_{2}\left(e^{\theta_{1}(s-t)}-1\right)+\theta_{1}}\right)^{\theta_{3}} ; B(t, s)=\left(\frac{e^{\theta_{1}(s-t)}-1}{\theta_{2}\left(e^{\theta_{1}(s-t)}-1\right)+\theta_{1}}\right)
$$

and

$$
\theta_{1}=\sqrt{(\kappa+\lambda)^{2}+2 \sigma^{2}}, \quad \theta_{2}=\frac{(\kappa+\lambda)+\theta_{1}}{2}, \quad \theta_{3}=\frac{2 \kappa \bar{r}}{\sigma^{2}}
$$

Let $F$ denote the value of the investment opportunity with a lifetime of length $T$ and starting at a future time $t$. The payoff from investing at the unknown future time $t$ is given by $V\left(r_{t}, t, T\right)-I$, where $V$ is defined in equation (5) and $I$ denotes the sunk cost of the investment project made at time $t$. Our aim is to maximize the conditional expected net present value (NPV) evaluated at present time, denoted as 0 , that is

$$
F\left(r_{0}\right)=\sup _{\widetilde{\tau}} E_{0}^{\mathbb{Q}}\left[\exp \left(-\int_{0}^{\widetilde{\tau}} r_{u} d u\right)\left(V\left(r_{\widetilde{\tau}}, \widetilde{\tau}, T\right)-I\right)\right]
$$

such that $\widetilde{\tau}$ is a (random) stopping time for the stochastic process driving equation (2). As long as the relationship between $V$ and $r$ is inverse, the optimal exercise policy will be investing if $r_{0} \leq r^{*}$ while waiting otherwise. The parameter $r^{*}$ 
denotes the threshold rate - the value for which the owner of the project is indifferent between waiting or exercising immediately by incurring the set-up costs of the project - which is a free boundary condition. As Dixit and Pindyck (1994) show, equation (10) is an optimal stopping problem in continuous time. Since the investment opportunity $F$ yields no cash flows up to the period where the investment is undertaken, the only return from holding it is its capital appreciation. Hence, in the continuation region $\left(r_{0}>r^{*}\right)$ the Bellman equation leads to the following second order ordinary differential equation (ODE):

$$
\frac{\sigma^{2}}{2} r F_{r r}+(\kappa+\lambda)\left(\frac{\kappa}{\kappa+\lambda} \bar{r}-r\right) F_{r}-r F=0
$$

In addition, $F(r)$ must satisfy three boundary conditions. First, the terminal condition $F(r)=0$ as $r \rightarrow \infty$ that will help us to set one of the two constants in the solution of (11). Two further conditions are also required to set the other constant plus the threshold rate. These are the so called 'value matching' and 'smooth pasting' conditions, given respectively by $F\left(r^{*}\right)=V\left(r^{*}\right)-I$ and $F_{r}\left(r^{*}\right)=V_{r}\left(r^{*}\right)$. These conditions mean that both, the values and the slopes of the two functions $F$ and $V$, match at the boundary $r^{*}$. Then, we obtain the following result ${ }^{1}$ :

Proposition 1 The general solution to the second order ODE defined in (11) is given by

$$
F(r)=e^{\nu r}\left\{C_{1} M(a, b ; x(r))+C_{2} x(r)^{1-b} M(a-b+1,2-b ; x(r)\}\right.
$$

where $M(a, b ; x)$ denotes the confluent hypergeometric function which has the series

\footnotetext{
${ }^{1}$ The following proposition would have a similar format for other mean reverting processes that can be found in Table 5 of Ingersoll and Ross (1992). We have obtained some of them and they are available from the authors upon request.
} 


$$
M(a, b ; x)=1+\frac{a}{b} x+\frac{a(a+1)}{b(b+1)} \frac{x^{2}}{2 !}+\ldots+\frac{a(a+1) \ldots(a+(k-1))}{b(b+1) \ldots(b+(k-1))} \frac{x^{k}}{k !}+\ldots
$$

and

$$
\begin{gathered}
a=\frac{\kappa \bar{r}}{\sigma^{2}}\left(1-\frac{\kappa+\lambda}{\theta_{1}}\right), b=\theta_{3}, \nu=\frac{(\kappa+\lambda)-\theta_{1}}{\sigma^{2}}, x(r)=\left(\frac{2 \theta_{1}}{\sigma^{2}}\right) r ; \\
C_{2}=-C_{1} \frac{\Gamma(b)}{\Gamma(a)} \frac{\Gamma(1+a-b)}{\Gamma(2-b)}
\end{gathered}
$$

where $\Gamma(\cdot)$ denotes the gamma function and $C_{1}$ is an arbitrary constant.

Proof. See Appendix.

Both $r^{*}$ and $C_{1}$ are obtained as the solution of the two non-linear equation system given by the last two boundary conditions mentioned above. Note that Proposition 1 is an alternative form of presenting the result appearing in Table 5 of Ingersoll and Ross (1992) for several stochastic processes driving the interest rate $r^{2}$. This table is constructed by making use of the properties of conditional expectations so that equation (10) can be expressed as the product of an expected stochastic discount factor $(\mathrm{SDF})$

$$
\Phi\left(r_{0}, r^{*}\right)=E_{0}^{\mathbb{Q}}\left[\exp \left(-\int_{0}^{t^{*}} r_{u} d u\right)\right]
$$

and the NPV of the project for a given $r^{*}, V\left(r^{*}, t^{*}, T\right)-I$. Then, $\Phi\left(r_{0}, r^{*}\right)$ satisfies an ODE analogous to equation (11) but subject to the boundary conditions

$$
\lim _{r_{0} \rightarrow \infty} \Phi\left(r_{0}, r^{*}\right)=0 \quad \text { and } \quad \Phi\left(r^{*}, r^{*}\right)=1
$$

It is verified that $\Phi(\cdot)$ is a decreasing function of $r_{0}$ while increasing for $r^{\star} 3$.

\footnotetext{
${ }^{2}$ There appears to be a typo in their solution of the stochastic discount factor when $r$ evolves according to equation (2).

${ }^{3}$ This follows from the general solution for the $\operatorname{SDF}$ given by $\Phi\left(r_{0}, r^{*}\right)=\frac{\phi\left(r_{0}\right)}{\phi\left(r^{*}\right)}$ where $\phi(\cdot)$ is
} 
Figure 1 exhibits the typical shape of the functions describing the project value in terms of the actual short rate $r_{0}$. The dashed line shows the NPV of the project while the solid line displays the project value incorporating the waiting option. For those interest rates values lower than the threshold rate, the owner of the project should invest now, otherwise waiting would be optimal.

As Figure 1 illustrates, the internal rate of return (IRR) - or breakeven rateis consistently higher than the threshold rate. For the particular values of the parameters illustrated in the figure, $\bar{r}=0.03, \kappa=0.45, \lambda=0.0, \sigma=0.15, T=30$ years and $I=5$, the IRR equals 0.7967 whereas the value of $r^{*}$ is 0.1073 .

It is also interesting to obtain the length of the waiting period until the investment option is exercised, or in other words, finding out the time needed for the actual interest rate to revert to the threshold rate. It does require to calculate the first hitting time density of the level $r^{*}$ starting from $r_{0}>r^{*}$. For $r_{0}-r^{*}$ equals $500 \mathrm{bp}$ (0.05), the mean hitting time ${ }^{4}$ for the previous set of parameter values is about 0.6797 years or 8.3 months. This implies a probability of 0.3117 that the hitting time will be greater than that average.

To end this section, note that the dynamics of $r$ studied by Ingersoll and Ross (1992) — which is nested in equation (2) by setting $\kappa=0$ - yields an expression for the value of the project with the option of waiting which is nested in equation (12) when $\kappa=0$.

a decreasing function whose specific form depends on the particular stochastic process driving interest rates. For more details, see Table 5 in Ingersoll and Ross (1992).

${ }^{4}$ See Linetsky (2004), specifically equations (23) and (24). 
Corollary 2 The solution to the second order ODE in (11), when $\kappa$ is set to zero in equation (2), is given by

$$
F(r)=C_{1} e^{\nu r}
$$

Proof. It follows easily by taking the limit of equation (12) as $\kappa \rightarrow 0$ and noticing that

$$
\lim _{\kappa \rightarrow 0} \frac{\Gamma(b) \Gamma(1+a-b)}{\Gamma(a) \Gamma(2-b)}=\frac{a}{b}
$$

for $a$ and $b$ evaluated at $\kappa=0$.

As can be checked, equation (6) in Ingersoll and Ross (1992) is essentially the same as ours. Of course, this solution can also be obtained directly from the ODE defined in equation (11) by setting $\kappa=0$.

\section{Sensitivity Analysis}

As proposition 1 makes clear, the value of $r^{*}$ depends on several parameters. In general, it is extremely difficult to obtain analytical results and a sensitive analysis is performed to describe the likely effects of changes in the parameters. In this section we shall take $\lambda=0$ as our benchmark value, leaving for the next section the impact of changes in the market risk parameter.

To guide our intuition for interpreting the results in the sensitivity analysis, recall that the value of the project at any time can be decomposed into the SDF and the project NPV evaluated at $r^{*}$. Intuitively, anything that raises the NPV will increase the opportunity cost of waiting and hence, generate an upward pattern for $r^{*}$. That is, the project's owner will take the decision to invest for relatively 'higher' interest rates. Similarly, anything that raises the SDF value will increase the benefits of waiting since the PV of all future cash flows becomes higher. So, the decision to invest now will be taken for relatively 'lower' interest rates. All this 
seems fairly intuitive but we need to ascertain the likely effects on both NPV and SDF of changes in the CIR parameters.

Cox, Ingersoll and Ross (1985) state that a higher (lower) value of $\sigma(\bar{r})$ has the effect of rising the discount bond price at any maturity. This behavior is also reproduced in equation (5). Thus, the effect of either increases in $\sigma$ or decreases in $\bar{r}$ is rising both NPV and SDF. For the likely effect on NPV and SDF of changes in $\kappa$, it turns out that increases in this parameter rises both for low values of $\sigma$. But as $\sigma$ becomes higher, this effect is reversed.

The remarkable result is that changes in the CIR parameters have the same impact on NPV and SDF but opposite effects on $r^{*}$.

Ingersoll and Ross (1992) are concerned with the effect that interest rate uncertainty has on the investment rate and, to this end, they examine the impact that higher values of $\sigma$ have on $r^{*}$. In this regard, they confine themselves to the case of no mean reversion or $\kappa=0$. They find that a higher value of $\sigma$ lowers $r^{*}$ for $\lambda=0$. We also obtain a similar relationship for the general case of mean reversion. Since $r^{*}$ falls with $\sigma$, the SDF effect appears to outweight the NPV effect as shown in Figure 2 for several values of $\kappa$ (panel A) and $\bar{r}$ (panel B). We have also plotted the no mean reversion case in panel A. The fall in $r^{*}$ becomes more pronounced under no mean reversion when $\sigma$ increases. In this figure we find some evidence that higher values of $\kappa$ raises $r^{*}$, which is consistent with a higher weight of the SDF effect. Further, as panel B makes clear, the curve $\left(\sigma, r^{*}\right)$ shifts upwards with higher values of $\bar{r}$.

In Figure 3 increases in $\bar{r}$, while keeping fixed the remaining parameters $\kappa$ and $\sigma$, yields an inverted 'U-shaped' curve for $r^{*}$. This behavior is displayed for several 
values of the the parameters $\kappa$ in panel $\mathrm{A}$ and $\sigma$ in panel $\mathrm{B}$. The shifts in the curve $\left(\bar{r}, r^{*}\right)$ due to changes in $\sigma$ and $\kappa$ are in agreement with our previous interpretation so it does not deserve further comments.

In Figure 4 increases in $\kappa$ tend to increase $r^{*}$ suggesting that the SDF effect prevails over the NPV effect. Panel A illustrates this for several values of $\sigma$ except for the lowest. Recall that, for low values of $\sigma$, a higher value of $\kappa$ tend to increase, in particular, SDF and a higher SDF calls for a lower $r^{*}$. Furthermore, higher values of $\sigma$ shifts downwards the curve $\left(\kappa, r^{*}\right)$. Panel B also illustrates that increases in $\bar{r}$ shifts the curve $\left(\kappa, r^{*}\right)$ upwards and this increase growths as the value of $\kappa$ rises.

Finally, according to what has been described as the NPV effect, a larger value of $T$ increases the threshold rate $r^{*}$. This effect is the opposite when the value of $I$ increases since this means a reduction of the NPV. These effects are shown, respectively, in panels $\mathrm{A}$ and $\mathrm{B}$ of Figure 5 .

\section{Market Price of Risk}

First, we show that changes in the parameter of risk aversion are equivalent to changes in the parameter underlying the market price of risk, namely the parameter $\lambda$ in equation (4). And second, we study the impact of $\lambda$ on the behavior of $r^{*}$. 


\subsection{Framework}

Notice that $\gamma(r, t)$, defined in equation (4), is the excess bond expected return $\left(\mu_{P}-r\right)$ per unit of volatility $\left(\sigma_{P}\right)$ or Sharpe ratio. Let $\Lambda$ denote the SDF, which is directly related to the consumption marginal utility. Then, $\mu_{P}-r$ can be written in terms of the covariance of the bond return and SDF as

$$
\mu_{P}-r \equiv \frac{1}{d t} E_{t}^{\mathbb{P}}\left(\frac{d P}{P}\right)-r=-\frac{1}{d t} E_{t}^{\mathbb{P}}\left(\frac{d P}{P} \frac{d \Lambda}{\Lambda}\right)
$$

where $P(r, t, s)$ is the price of a zero-coupon bond satisfying the PDE in equation (6), see Cochrane (2001). If the interest rate is driven, under the real measure $\mathbb{P}$, by the general stochastic process

$$
d r=\mu_{r}(r, t) d t+\sigma_{r}(r, t) d W^{\mathbb{P}}
$$

we can use Ito's Lemma to obtain the following stochastic differential equation (SDE) for this bond price:

$$
d P / P=\mu_{P}(r, t) d t+\sigma_{P}(r, t) d W^{\mathbb{P}}
$$

where $\sigma_{P}(r, t) \equiv-\frac{\partial P}{\partial r} \frac{\sigma_{r}(r, t)}{P}$. By the other hand, $\Lambda$ in equation (19) satisfies the following general SDE:

$$
d \Lambda / \Lambda=-r d t-\sigma_{\Lambda}(r, t) d W^{\mathbb{P}}
$$

such that $E_{t}^{\mathbb{P}}(d \Lambda / \Lambda)=-r_{t} d t$ and the diffusion term $\sigma_{\Lambda}(r, t)$ depends, in particular, on the dynamics of the interest rate given in equation (20). If we compute the righthand side of equation (19), then $\mu_{P}-r=\sigma_{\Lambda} \sigma_{P}$ and $\gamma=\sigma_{\Lambda}$. This means that positive premiums, that is $\mu_{P}-r>0$, will arise if $\sigma_{\Lambda}>0$. Since $\sigma_{\Lambda}$ is the same for bonds of all maturities, a positive value of $\sigma_{\Lambda}$ implies that long bond expected returns are higher than short rates. Hence, for the CIR case or equation $(1)-\mu_{r} \equiv \kappa(\bar{r}-r)$ 
and $\sigma_{r} \equiv \sigma \sqrt{r}$ in equation (20) —, the risk price parameter $\lambda$ in equation (4) must be negative since $\sigma_{\Lambda}=-\lambda \sqrt{r} / \sigma>0$. The introduction of the SDF in equation (22) also leads us to value these bonds, that is, $P\left(r_{t}, t, s\right)=E_{t}^{\mathbb{P}}\left(\Lambda_{t+s} / \Lambda_{t}\right)$.

Let $\Lambda_{t} \equiv e^{-\beta t} u^{\prime}\left(c_{t}\right)$, where $\beta$ captures impatience for the subjective discount factor $e^{-\beta t}, c_{t}$ denotes consumption at date $t$ and $u^{\prime}(\cdot)$ is the marginal utility. By applying Ito's lemma, we can rewrite the SDF in equation (22) as

$$
d \Lambda / \Lambda=-\beta d t+\frac{c u^{\prime \prime}(c)}{u^{\prime}(c)} \frac{d c}{c}+\frac{1}{2} \frac{c^{2} u^{\prime \prime \prime}(c)}{u^{\prime}(c)}\left(\frac{d c}{c}\right)^{2}
$$

where $\xi \equiv-c u^{\prime \prime}(c) / u^{\prime}(c)$ denotes the coefficient of relative risk aversion (RRA). Since the correlation between both Wiener processes of $c$ and $\Lambda\left(\rho_{c \Lambda}\right)$ is less or equal than 1 , it holds that $\sigma_{\Lambda} \leq \xi \sigma_{c}$ where $\sigma_{c}$ is the volatility of the SDE of $d c / c$. If we assume the standard power utility for consumption, that is, $u(c)=c^{1-\eta} /(1-\eta)$ then $\eta$ becomes the RRA coefficient or $\xi=\eta$. In short, there is a direct relationship between $\sigma_{\Lambda}$ and $\eta$. Hence, analyzing the effects of changes in $\sigma_{\Lambda}$-through changes in $\lambda$ - on $r^{*}$ is equivalent to examining the effects of changes in $\eta$ on $r^{*}$. For instance, considering a power utility function, a value of one for $\rho_{c \Lambda}$ and the Vasicek (1977) model for the interest rate $-\mu_{r} \equiv \kappa(\bar{r}-r)$ and $\sigma_{r} \equiv \sigma$ in equation (20) which implies a constant value for $\sigma_{\Lambda}$ in equation (22) as Cochrane (2001) shows, then $\sigma_{\Lambda}=\eta \sigma_{c}$.

\subsection{Sensitivity Analysis of $\lambda$}

The market risk parameter, $\lambda$, exhibits an implicit negative sign to guarantee a positive risk premium. A negative value of $\lambda$ means that long term rates are high relative to short rates. As it was discussed before, we can distinguish two opposite effects: a higher absolute value, or size, of $\lambda$ leads to a lower NPV. This generates a downward pattern for $r^{*}$ since it reduces the opportunity cost of waiting as related to 
a higher value of $\eta$. As it is well known, a higher value of $\eta$ (higher size of $\lambda$ ) implies a preference for less uncertain results realized in the near future in comparison with potentially higher uncertain ones. By the other hand, and at the same time, a higher size of $\lambda$ also causes a fall in the SDF. This is because investors demand a higher return from risky investments, increasing the interest rate at any maturity and reducing the value of the SDF. Now, the benefits of waiting are lower and $r^{*}$ tends to grow.

Panel A in Figure 6 exhibits the $\left(\lambda, r^{*}\right)$ relationship under no mean reversion. We observe that, for small sizes of $\lambda$, the SDF effect prevails and $r^{*}$ at first increases. However, as the size of $\lambda$ increases, the NPV effect becomes prevalent and $r^{*}$ begins to fall. In Ingersoll and Ross (1992), it is not clear whether a higher size of $\lambda$ leads to a lower $r^{*}$. What they clearly state is that the difference between the IRR of the project and the threshold rate falls with higher magnitudes of $\lambda$. This result (not reported here) also holds within our framework independently of whether $r^{*}$ rises or falls. Ingersoll and Ross (1992) also find that a higher value of $\sigma$ reduces $r^{*}$. We also obtain this result in panel A.

With mean reversion, the impact that changes in $\lambda$ has on $r^{*}$ shows a more pronounced humped shape as exhibited in panel B. Now the SDF effect exceeds the NPV effect for comparatively lower magnitudes of $\lambda$. A possible interpretation is that now future interest rates tend to be close to $\bar{r}$, exhibiting a lower variability which might be associated with higher values of $r^{*}$.

In short, under the no mean reversion case, we might conclude that a higher market price of risk, and hence a higher value of the RRA parameter, lowers $r^{*}$. However, a lower value of $\sigma$ also means a higher market price of risk and this raises $r^{*}$ instead. Nevertheless, under mean reversion, there is some evidence that a higher 
market price of risk implies a higher value of $r^{*}$. This result is also obtained by Alvarez and Koskela (2006b).

\section{Conclusions}

We examine the characteristics of an optimal irreversible investment policy when the source of uncertainty comes from interest rates. Special attention is paid to the role that risk aversion plays on the threshold rate that guides the investment decision. The one-dimensional stopping problem proposed here uses the well-known CIR process. We first obtain a closed-form solution for the option to invest and show how it is related to the seminal work of Ingersoll and Ross (1992). We also carry out an extensive sensitive analysis about how the threshold rate is affected by different values of the parameters underlying the CIR model. We find that a higher relative risk aversion leads to higher threshold rates when the interest rate is mean reverting. This effect is reversed, however, when there is no mean reversion.

\section{References}

[1] Abramowitz, M. and I.A. Stegun, 1972. Handbook of Mathematical Functions with Formulas, Graphs and Mathematical Tables. Dover, New York.

[2] Alvarez, L.H.R. and E. Koskela, 2006a. Irreversible investment under interest rate volatility: Some generalizations. The Journal of Business 79, 2, 623-644.

[3] Alvarez, L.H.R. and E. Koskela, 2006b. Does risk aversion accelerate optimal forest rotation under uncertainty?. The Journal of Forest Economics 12, 171184.

[4] Cochrane, John H., 2005. Asset Pricing. Princeton University Press. 
[5] Cox, J.C., J.E. Ingersoll and S.A. Ross, 1985. A theory of the term structure of interest rates. Econometrica 53, 385-407.

[6] Dixit, A.K. and R.S. Pindyck, 1994. Investment under Uncertainty. Princeton University Press.

[7] Ingersoll, J.E. and S.A. Ross, 1992. The value of waiting to invest: Investment and uncertainty. Journal of Business 65, 1, 1-29.

[8] Linetsky, V., 2004. Computing hitting time densities for CIR and OU diffusions: applications to mean-reverting models. Journal of Computational Finance 7, 4, $1-22$.

[9] McDonald, R. and D. Siegel, 1986. The value of waiting to invest. The Quarterly Journal of Economics 101, 4, 707-727.

[10] Polyanin, A.D. and V.F. Zaitsev, 2003. Handbook of Exact Solutions for Ordinary Differential Equations. Chapman and Hall, CRC.

[11] Vasicek, O., 1977. An equilibrium characterization of the term structure. Journal of Financial Economics 5, 177-188. 


\section{Appendix}

Let us multiply equation (11) by $\exp (-\nu r)$ to get

$$
r e^{-\nu r}\left\{\frac{\sigma^{2}}{2} F^{\prime \prime}(r)-(\kappa+\lambda) F^{\prime}(r)-F(r)\right\}+\kappa \bar{r} F^{\prime}(r) e^{-\nu r}=0 .
$$

and compute the roots corresponding to the term in brackets in (24)

$$
\frac{\sigma^{2}}{2} \nu^{2}-(\kappa+\lambda) \nu-1=0
$$

to obtain: $\nu_{1}=\frac{(\kappa+\lambda)+\theta_{1}}{\sigma^{2}}>0$ and $\nu_{2}=\frac{(\kappa+\lambda)-\theta_{1}}{\sigma^{2}}<0$ where $\theta_{1}$ is defined as in (14). Now, let $J(r)=F(r) e^{-\nu r}$. Select $\nu_{2}$, write equation (24) in terms of $J(r)$ and, finally, add and subtract $\nu_{2} \kappa \bar{r} J(r)$ from this equation. As a result, equation (24) can be rewritten as

$$
r J^{\prime \prime}(r)+\left(\frac{2 \kappa \bar{r}}{\sigma^{2}}-2 \frac{\theta_{1}}{\sigma^{2}} r\right) J^{\prime}(r)+2 \frac{\kappa \bar{r} \nu_{2}}{\sigma^{2}} J(r)=0 .
$$

Let $x=2 \frac{\theta_{1}}{\sigma^{2}} r$ and $A(x)=J(r)$, so that we can write equation (26) as the 'confluent hypergeometric equation'

$$
x A_{x x}(x)+(b-x) A_{x}(x)-a A(x)=0
$$

where $a>0$ and $b>0$ are defined in (14). Its general solution has the form: ${ }^{5}$

$$
C_{1} M(a, b ; x)+C_{2} x^{1-b} M(a-b+1,2-b ; x)
$$

for $M(a, b ; x)$ denoting the confluent hypergeometric function defined in (13). $C_{1}$ and $C_{2}$ are arbitrary constants to be determined with the appropriate boundary conditions. If we multiply equation (28) by $\exp \left(\nu_{2} r\right)$ and reverse the change of variables, we shall recover the general expression for $F(r)$ defined in (12) which is the solution to equation (11). Using the boundary condition $F(r)=0$ as $r \rightarrow \infty$ and considering that ${ }^{6}$ as $x \rightarrow \infty$ $(x>0)$ :

$$
M(a, b, x)=\frac{\Gamma(b)}{\Gamma(a)} e^{x} x^{a-b}\left[1+O\left(x^{-1}\right)\right]
$$

where the above notation means that both $M(a, b, x)$ and $\frac{\Gamma(b)}{\Gamma(a)} e^{x} x^{a-b}$ are asymptotically equal for large values of $x$. By inserting equation (29) into (28), we get (15) which completes the proof.

\footnotetext{
${ }^{5}$ See equation 70 from page 220 in Polyanin and Zaitsev (2003).

${ }^{6}$ See Abramowitz and Stegun (1972) on page 504.
} 


\section{Figures}

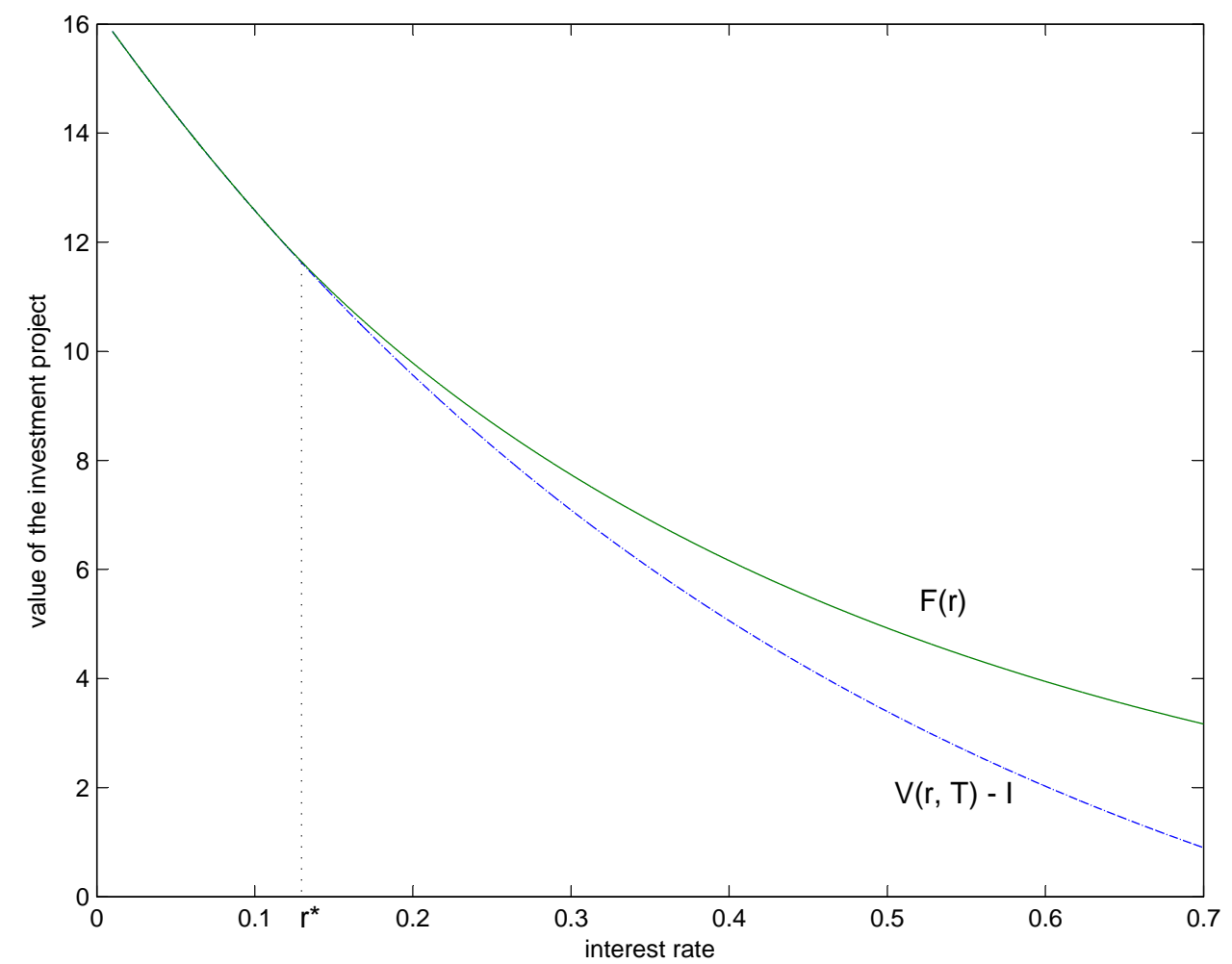

Figure 1: Value of the investment project including the waiting option (solid line) and without it (dashed line). Parameter values: $\bar{r}=0.03, \kappa=0.45, \lambda=0.0$, $\sigma=0.15, T=30, I=5$ 


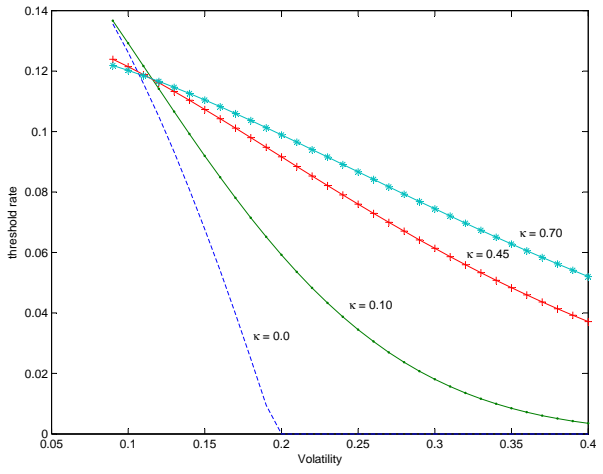

A. Parameter values: $\bar{r}=0.03, T=30$, $I=5$

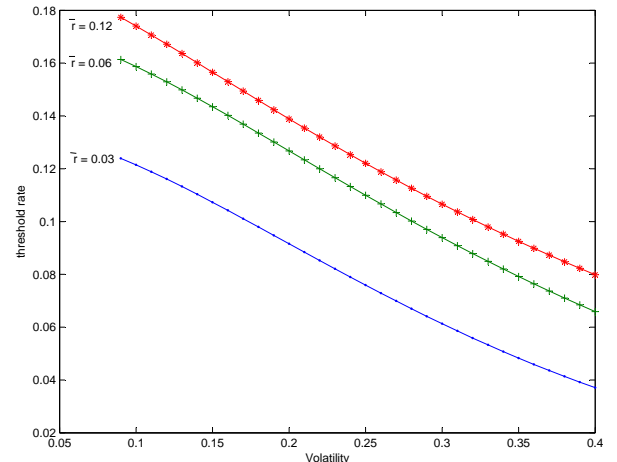

B. Parameter values: $\kappa=0.45, T=30$, $I=5$

Figure 2: Impact of changes in the volatility parameter $(\sigma)$.

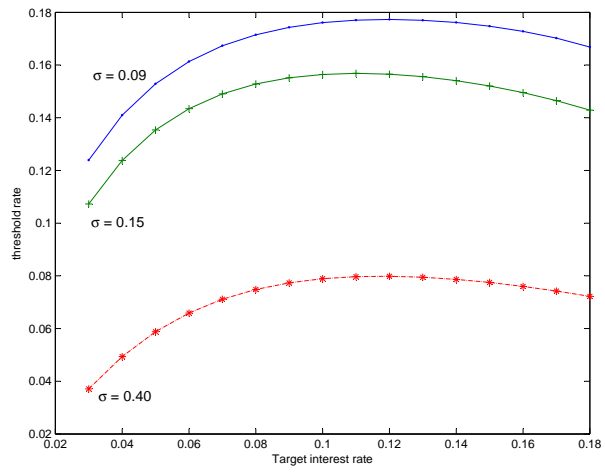

A. Parameter values: $\kappa=0.45, T=30$, $I=5$

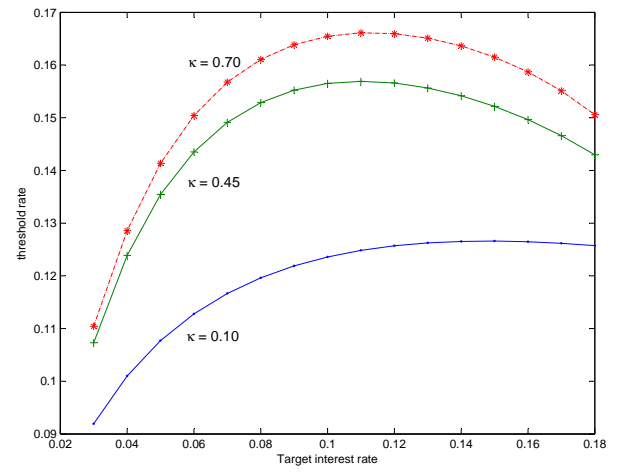

B. Parameter values: $\sigma=0.15, T=30$, $I=5$

Figure 3: Impact of changes in the target interest rate $(\bar{r})$.

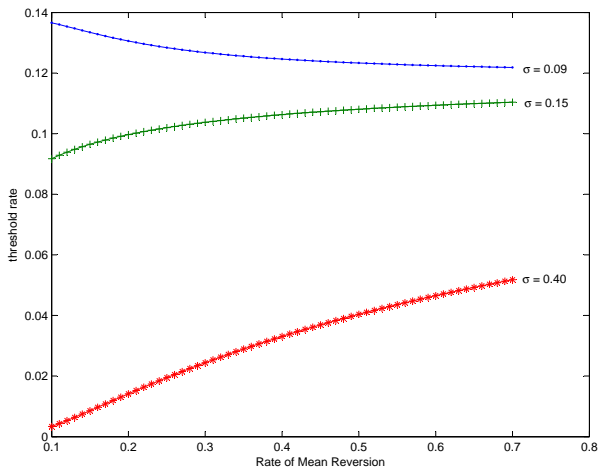

A. Parameter values: $\bar{r}=0.03, T=30$, $I=5$

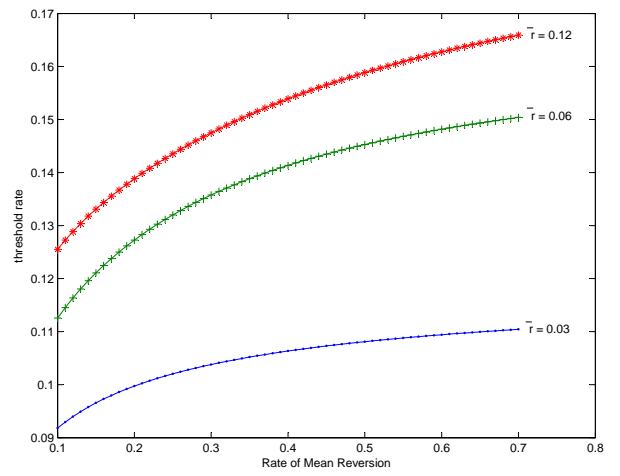

B. Parameter values: $\sigma=0.15, T=30$, $I=5$

Figure 4: Impact of changes in the speed of mean reversion parameter $(\kappa)$. 


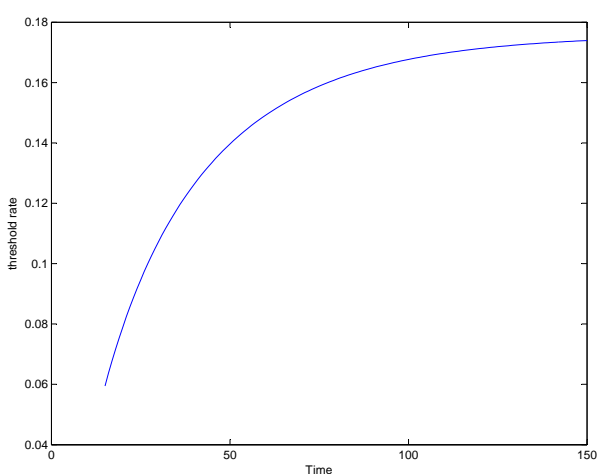

A. Parameter values: $\bar{r}=0.03 ; \kappa=0.45$, $\sigma=0.15, I=5$

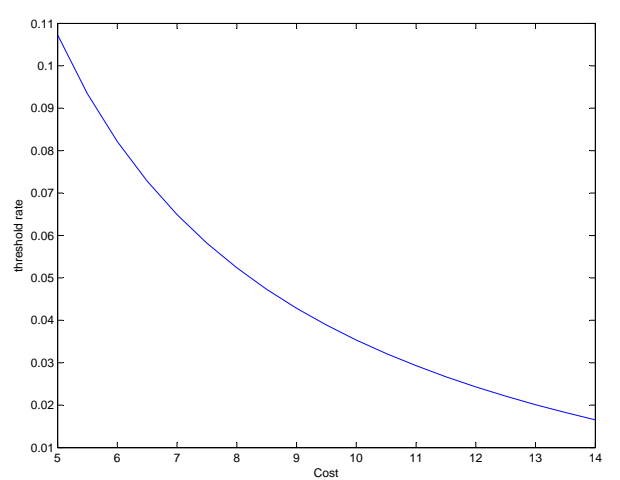

B. Parameter values: $\bar{r}=0.03 ; \kappa=0.45$, $\sigma=0.15, T=30$

Figure 5: Impact of changes in the maturity of the investment project $(T)$ and the cost of investment $(I)$.

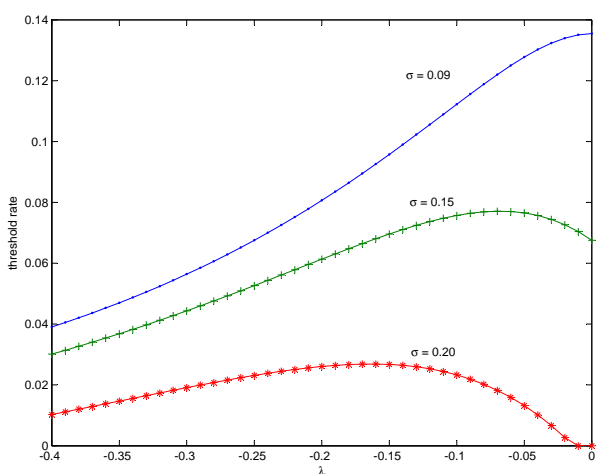

A. Parameter values: $\kappa=0, T=30$, $I=5$

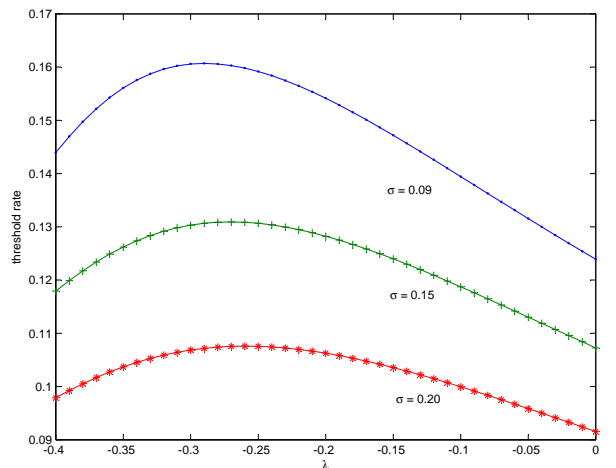

B. Parameter values: $\bar{r}=0.03 ; \kappa=0.45$, $T=305, I=5$

Figure 6: Impact of changes in the market risk parameter $(\lambda)$. 